\title{
A pilot study on a new anchoring mechanism for surgical applications based on mucoadhesives
}

\author{
SELENE TOGNARELLI ${ }^{1}$, VIRGINIA PENSABENE ${ }^{1,2}$, SARA CONDINO $^{3}$, \\ PIETRO VALDASTRI ${ }^{1}$, ARIANNA MENCIASSI ${ }^{1}$, ALBERTO AREZZO $^{4}$, PAOLO DARIO $^{1,2}$ \\ ${ }^{1}$ CRIM Lab, Scuola Superiore Sant'Anna, Pisa, Italy, ${ }^{2}$ Italian Institute of Technology (IIT), Center for \\ MicroBioRoboticsIIT@SSSA, Pontedera, Italy, ${ }^{3}$ ENDOCAS Center for Computer Assisted Surgery, University of Pisa, \\ Pisa, Italy, and ${ }^{4}$ Centre of Minimally Invasive Surgery, University of Torino, Italy
}

\begin{abstract}
In order to minimize the invasiveness of laparoscopic surgery, different techniques are emerging from research to clinical practice. Whether the incision is performed on the outside - as in Single Port Laparoscopy (SPL) - or on the inside - as in Natural Orifice Transluminal Endoscopic Surgery (NOTES) - of the patient's body, inserting and operating all the instruments from a single access site seems to be the next challenge in surgery. Magnetic guidance has been recently proposed for controlling surgical tools deployed from a single access. However, the exponential drop of magnetic field with distance makes this solution suitable only for the upper side of the abdominal cavity in nonobese patients. In the present paper we introduce a polymeric anchoring mechanism to lock surgical assistive tools inside the gastric cavity, based on the use of mucoadhesive films. Mucoadhesive properties of four formulations, with different chemical components and concentration, are evaluated by using both in vitro and ex vivo test benches on porcine stomach samples. Hydration of mucoadhesive films by contact with the aqueous mucous layer is analyzed by means of in vitro swelling tests, whereas optimal preloading conditions and adhesion performances, in terms of detachment force, supported weight and size are investigated ex vivo. Mucoadhesion is observed with all the four formulations. For a contact area of $113 \mathrm{~mm}^{2}$, the maximum normal and shear detachment forces withstood by the adhesive film are $2,6 \mathrm{~N}$ and $1 \mathrm{~N}$ respectively. These values grow up to $12,14 \mathrm{~N}$ and $4,5 \mathrm{~N}$ when the contact area increases to $706 \mathrm{~mm}^{2}$. Lifetime of the bonding on the inner side of the stomach wall was around two hours. Mucoadhesive anchoring represents a fully biocompatible and safe approach to deploy multiple assistive surgical tools on mucosal tissues by minimizing the number of access ports. This technique has been quantitatively assessed ex vivo for anchoring on the inner wall of the gastric cavity or in gastroscopic surgery. By properly varying the chemical formulation, this approach can be extended to other cavities of the human body.
\end{abstract}

Key words: Mucoadhesive films, anchoring system, minimally invasive surgery, single port laparoscopy, natural orifices transluminal surgery

\section{Introduction}

Laparoscopy has revolutionized the methods used by surgeons in traditional procedures, producing important advantages in terms of decreased postoperative pain, improved cosmetics and reduced hospitalization. Recently, there has been an impetus in the surgical community to further reduce the invasiveness of laparoscopic surgery, designing and developing new instrumentation and technologies. To achieve this goal, surgeons proposed to limit the number of abdominal incisions (Single Port Laparoscopy - SPL or LaparoEndoscopic Single-Site surgery - LESS (1)) or to eliminate them completely (Natural Orifice Transluminal Endoscopic Surgery - NOTES (2)). However, by reducing the number of trocars/incisions, and thus introducing the current endoscopes and instruments together at a single site, several technical restrictions arise. Among these, the most critical issues are a limited triangulation, poor ergonomics, limited

Correspondence: S. Tognarelli, CRIM Lab, Scuola Superiore Sant́Anna, Piazza Martino della Libertà, 33-56127 Pisa, Italy.

E-mail: selene.tognarelli@mail.crim.sssup.it 
visual axis and field of view, and internal and external collision of instruments (2). In the case of SPL or NOTES, using a single trocar or a flexible endoscope to deploy Assistive Internal Surgical Instruments (AISI) can overcome some of these hurdles.

For laparoscopic interventions, miniature magnetically guided devices that fit entirely inside the abdomen were presented in $(3,4)$. They consist of instruments which are introduced through a single trocar into the abdominal cavity and are then stabilized on the peritoneum by external handheld magnets. Trans-abdominal magnetic anchoring and guidance systems (MAGS) for minimally invasive surgery were demonstrated in laparoscopic procedures on animals (2), introducing a camera and two tissue retractors through a standard $12 \mathrm{~mm}$ trocar port. The maximum weight of a single AISI was $45 \mathrm{~g}$, which was fully supported by the transabdominal magnetic link. From a technical standpoint, the most advanced system exploiting magnetic fixation and positioning consists in a peritoneum-mounted imaging robot, as reported by Oleynikov et al. (5). It is a stationary outer tube of $21 \mathrm{~mm}$ in diameter, with a rotating inner tube that houses the lens, a camera board, and three micromotors, for a total weight of $75 \mathrm{~g} \mathrm{(6).} \mathrm{An} \mathrm{improved}$ prototype of this robotic camera, $12 \mathrm{~mm}$ in diameter, is described by Canes and coworkers (7).

However, relying on magnetic field for device anchoring and stabilization introduces a set of limitations still far from being solved. The coupling strength decreases exponentially with respect to the distance between the two magnets, thus limiting potential applications to the upper side of the abdominal cavity. Additionally, obese patients may not benefit from this approach due to the thick fat layer that acts as a spacer in between the external and the internal magnet. Furthermore, the operating area must not be crowded with magnets in order to prevent magnet-magnet interference and operator-magnet collisions. This issue typically limits the use of MAGS to one or two units maximum. When several units are used inside the abdominal cavity, two of them may occasionally come too close to each other and link together. If this happens, the procedure must be immediately converted to open surgery to retrieve the MAGS (3). Finally, as for magnetic resonance imaging (MRI), magnetic technology would be absolutely contraindicated for peacemaker holders, and harmful for patients with known metal foreign bodies or implanted metal orthopedic prostheses (8).

In order to overcome these limitations, while still maintaining the concept of introducing multiple tools from a single access, we propose a polymeric anchoring mechanism based on biocompatible polymeric bioadhesive films. This approach can be used to fix surgical assistive or diagnostic instrumentation to the inner wall of human cavities. Within the present study, we aim to achieve a proof of concept of the proposed strategy for the inner gastric cavity. AISI deployed in the stomach may be useful in the therapy of gastric cancer (9), in supporting funduplication procedures to treat gastroesophageal reflux disease (GERD) (10) and for bariatric surgery (11). Additionally, novel techniques, such as natural orifices transgastric surgery (12), may benefit from the use of purposely developed AISI attached to the inner gastric wall. Another interesting emerging technology where the proposed adhesion strategy can play a fundamental role is endoluminal robotic surgery. Harada et al. (13) deployed a modular robot via oral access in the gastric cavity to perform surgical operations. In this case the feet of the robot just push against the stomach wall, thus a stable adhesion is not guaranteed. Applying a film of mucoadhesive at the anchoring sites would improve stability of operation. Other devices reported in literature that may benefit from the work presented in this paper range from deployable $\mathrm{pH}$ or obscure bleeding sensors $(14,15)$, currently anchored to the lumen wall by surgical clips, to physiological transducers that can be used to monitor the status of a tissue during a surgical operation (16).

A bioadhesive can be defined as a synthetic or biological material capable of adhering onto a biological substrate or tissue (17). Bioadhesion is governed by several mechanisms, including swelling of the polymer and binding between film and tissue, by attractive molecular connections, Van der Waals, hydrogen and ionic bonds. The adhesive formulation can be modified in order to enable the attachment onto the wet membrane layers covering human organs, such as the peritoneum, or to the mucosal layer lining on the inner surface of the gastrointestinal tract. In this latter case, we usually refer to mucoadhesion.

This "mucoadhesion" is allowed by natural or synthetic hydrophilic macromolecules with high density of hydrogen bond-forming groups. They can be embedded in films or platelets in order to develop controlled drug carriers, able to locally release chemicals in the gastrointestinal area (18). In the work of Dodou et al. $(19,20)$, mucoadhesives were studied for generating high static friction between a colonoscopic device and the colonic wall.

Here we present a new application of these adhesive polymers to fix AISI, such as miniature cameras, or imaging and lighting robots (8) to the inner wall of the gastric cavity. The mucoadhesive properties were initially investigated by using small prototyping modules with a diameter of $12 \mathrm{~mm}$ that can be introduced into the stomach by flexible endoscopy through the oral access (21). Bearing in mind potential extension 
of the proposed technique to other human cavities, it is worthy to mention that the same modules are compatible with standard trocar for laparoscopic access, while SPL and LESS would allow the deployment of modules up to $35 \mathrm{~mm}$ in diameter (22).

In this paper, we analyze the adhesive properties of four different mucoadhesive chemical formulations and compare their performance by in vitro and ex vivo tests. A purposely developed test bench is presented in order to evaluate both the preload force and the time required for an efficient anchoring. The adhesion strength of these biocompatible mucoadhesive films is measured, presenting the detachment forces and debonding time that each formulation can afford. Finally, different dimensions for the mucoadhesive modules are tested in order to define a guideline for the design of a new generation of "mucoadhesive assistive internal surgical instruments". All the ex vivo tests of this pilot study were performed on the inner surface of the gastric cavity. Suggestions regarding how to approach different districts of the human body are outlined in the conclusions.

\section{Material and methods}

Adhesive properties of polymeric films with human mucous have already been investigated $(19,23)$. For our intended purpose, the mucoadhesive film should be designed considering that the physiological conditions change drastically in different human cavities, and in particular in the gastrointestinal tract regarding $\mathrm{pH}$, tissue morphology and thickness of mucous layer (19). In the present pilot study, four different kinds of mucoadhesive films were prepared by varying the method and formulation described by Dodou et al. (19), aiming to achieve adhesion on the inner side of the stomach. These were then compared with two sessions of in vitro and ex vivo tests.

\section{Mucoadhesive films preparation}

A $0.3 \% \mathrm{w} / \mathrm{w}$ and a $1 \% \mathrm{w} / \mathrm{w}$ mucoadhesive hydrogels were prepared by slowly sifting Carbopol CP 971P NF (a gift by Noveon Inc., Cleveland, OH, USA) into deionized water using a speed mixer. After that, the entire quantity of dry polymer was added. Stirring then continued for 15 additional minutes at moderate speed $(600 \mathrm{rpm})$ to prevent air entrapment into the dispersion. Afterwards, $<1 \mathrm{ml}$ of triethanolamine (TEA 33729, purchased from Sigma Aldrich, St. Louis, MO, USA) was added under mild stirring $(500 \mathrm{rpm})$ in order to neutralize the dispersion ( $\mathrm{pH}$ 6,9-7,2). In this way, hydrogels were obtained.
A $10 \% \mathrm{w} / \mathrm{w}$ polyvinylpyrrolidon (PVP, by SigmaAldrich), a 3\% w/w polypropylene glycol (PPG) and a $2 \% \mathrm{w} / \mathrm{w}$ polyetilenglycol (PEG) aqueous solutions were prepared under stirring at $800 \mathrm{rpm}$ for 15 minutes each. Finally, a $1 \%$ Pluronic PF127 (MW 12,600, Sigma Aldrich) aqueous solution was prepared and stirred for 15 minutes. At this stage of the procedure, two different hydrogel dispersions were prepared. The first one was obtained by mixing the $0,3 \% \mathrm{w} / \mathrm{w}$ Carbopol hydrogel with the PVP solution, PF127, and PEG or PPG aqueous solutions, while the second one was obtained by mixing the $1 \%$ w/w Carbopol hydrogel, with PVP, PF127, PPG or PEG solutions. Both dispersions were mixed under stirring at $800 \mathrm{rpm}$ for 15 minutes.

The produced dispersions were processed under vacuum at room temperature to remove the entrapped air and they were kept overnight at $4^{\circ} \mathrm{C}$ to complete hydration. The dispersions were returned to room temperature and poured into polystyrene Petri dishes. Next, the produced samples were dried in an oven at $38^{\circ} \mathrm{C}$ for $24 \mathrm{~h}$. After demoulding, the final thickness was $0,2 \mathrm{~mm}$ in all cases.

Table I shows, for each sample (S1, S2, S3, S4), the chemical elements and their concentration $(\% \mathrm{w} / \mathrm{w})$ in the final formulations.

\section{In vitro swelling study}

In general, mucoadhesion occurs in three stages, i.e. wetting, penetration and mechanical interlocking between polymer and mucous membrane (24-26). However, as most adhesion phenomena, the mucoadhesion process is still under debate in the scientific community, thus a single unified theory is not yet available (27).

Hydration of mucoadhesive films by contact with the aqueous mucous layer is a prerequisite for satisfactory mucoadhesion (28) and can be evaluated by measuring the water absorption capability of the polymer.

Table I. Chemical details for the four mucoadhesive formulations (S1, S2, S3, S4).

\begin{tabular}{lcccc}
\hline Ingredients & & & & \\
\hline Carbopol & $\mathrm{S} 1$ & $\mathrm{~S} 2$ & $\mathrm{~S} 3$ & $\mathrm{~S} 4$ \\
$P P G$ & $0,3 \%$ & $0,3 \%$ & $1 \%$ & $1 \%$ \\
$P V P$ & $3 \%$ & - & $3 \%$ & - \\
$P E G$ & $10 \%$ & $10 \%$ & $10 \%$ & $10 \%$ \\
$P F 127$ & - & $2 \%$ & - & $2 \%$ \\
\hline
\end{tabular}


The hydration behavior of the four polymeric formulations is determined by following the procedure described by Efentakis et al. (29). Briefly, fragments of each film, with an area of $95 \mathrm{~mm}^{2}$, were cut and $0,1 \mathrm{ml}$ of deionized water was added onto the surface. At fixed time intervals $(1,2,5,10$ and 15 minutes) the excess of water on the surface of swollen films was absorbed using blotting paper, and the samples were weighted.

The hydration percentages of the polymeric films were calculated according to the following equation (17):

$$
\text { Swelling Index }=\frac{W_{h}-W_{d}}{W_{d}}
$$

where $W_{d}$ and $W_{h}$ represent the weight of the dried and hydrated polymeric films, respectively.

Tests were repeated ten times for each sample and for each time interval and the final data were expressed as average value \pm standard deviation (S.D.).

\section{Ex vivo mucoadhesion tests}

A first set of experiments was carried out with a purposely developed setup, for the determination of preload force and time required for an efficient anchoring of AISI with mucoadhesion strategy and the evaluation of the normal and shear detachment forces and time that the mucoadhesive film can stand. In particular, the preload conditions can influence the penetration and mechanical interlocking between polymeric film and mucous membrane.

A second set of experiments was performed by using in part the same test bench for evaluating the relationship between the mucoadhesion and the dimension and weight of a generic AISI provided by mucoadhesive film.

Ex vivo experiments were carried out using freshly excised porcine stomach tissue gathered from the slaughterhouse on the days of the trials. Gastric specimens were gently cleaned in order to remove digestion debris, while preserving the mucous layer that protects the epithelial tissue.

The stomach model was selected because it presents a high quantity of mucous, which can be maintained in ex vivo conditions. More interestingly, the physiological conditions inside the stomach are the worst for the mucoadhesive film lifetime, because of the acid $\mathrm{pH}$ values (e.g. two to three in vivo conditions) (30). This means that if the films work properly in these conditions, they can perform even better in other sections of the gastrointestinal tract, where the $\mathrm{pH}$ is more neutral.

In all these tests, before starting the experiments, the $\mathrm{pH}$ value on the mucosal surface was measured by a pocket-sized $\mathrm{pH}$-meter with replaceable electrode (Hanna Instruments, Padova, Italy).

\section{Adhesion performance and preload requirements}

The porcine stomach was opened longitudinally and fixed, with the inner surface facing upwards on a Derlin plate $\left(14,5 \times 14 \mathrm{~cm}^{2}\right)$ by means of two rectangular constrains (Figure 1A).

The polymeric films were attached by cyanoacrylate adhesive to $12 \mathrm{~mm}$ diameter cylindrical rapidprototyping modules.

The film-covered module was placed in contact with the gastric tissue and the preload force ( 5 and $10 \mathrm{~N}$ ) was applied on the sample for a fixed time (one, three, five minutes) in order to ensure intimate contact between the tissue and the mucoadhesive sample.

A digital load cell (Alluris, FMI210, Freiburg, Germany) with $0,01 \mathrm{~N}$ resolution and $0-50 \mathrm{~N}$ measuring range was mounted on a servocontrolled linear slider (M-410CG, PI, Karlsruhe, Germany). The maximum stroke of the slider is $100 \mathrm{~mm}$, with an adjustable speed from $7 \mu \mathrm{m}$ up to
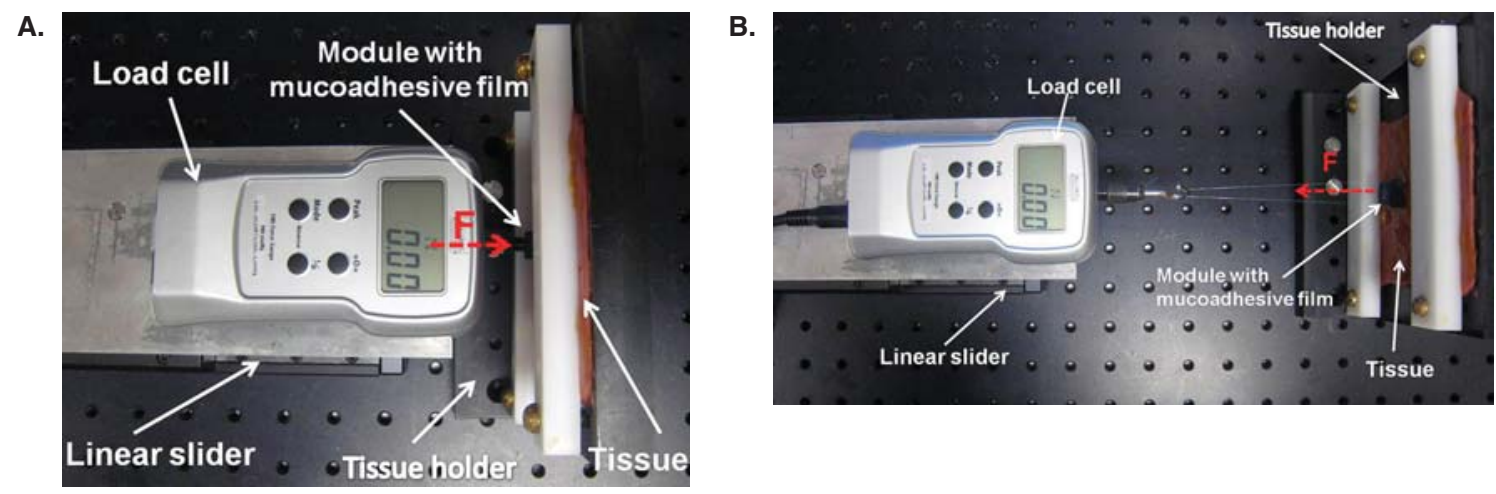

Figure 1. Experimental setup for preload application (A) and adhesive detachment force measurements (B). 
$1 \mathrm{~mm} / \mathrm{s}$. A proportional derivative (PD) controller was implemented in order to enable precise movement of the load cell during the measurements. As shown in Figure $1 \mathrm{~B}$, the load cell and the tissue holder were fixed so that a normal force can be applied to the film-covered module attached to the gastric specimen.

A continuous translational motion was imposed to the slider in the $\mathrm{Y}$ direction with a speed of $0,7 \mathrm{~mm} / \mathrm{s}$ and the mucoadhesive detachment force $\left(F_{D}\right)$ was determined for each chemical formulation.

Each measurement was repeated ten times on different areas of the gastric specimen. It is worthy to note that all the tissue samples were extracted from the same animal and the morphological differences between different gastric segments were assumed to be negligible.

The detachment force measurements for the four mucoadhesive formulations were aimed to select the best preload condition, in terms of force and time, and the best chemical formulation among the four proposed.

\section{Measurement of supported weight and size}

The mucoadhesive films were attached to cylindrical rapid-prototype modules and, based on the previous test session, the optimal preload condition was applied. As represented in Figure 2, the Derlin support with the tissue was turned upside-down and a weight holder was tied to the module. This allowed to vary the normal pulling load $(15,20$ and $25 \mathrm{~g}$ ), working against the mucoadhesive anchoring. The debonding time for the four mucoadhesive formulations under three different loading conditions was measured.

Based on the results obtained so far, the best performing polymeric formulation was attached to cylindrical modules having different diameters (i.e. 12, 15, 20 and $30 \mathrm{~mm}$ ), as represented in Figure 3, and $F_{D}$ was measured for each different module by using the same setup as described above. Preload force and time adopted in this experiment are the best ones assessed during the previous test sessions. Each test was performed ten times to achieve statistical relevance. The final goal of this experiment is to verify that, given that the adhesion is essentially a surface phenomenon, the detachment force is proportional to the mucoadhesive contact area. This would allow us to predict the required module diameter, given the AISI load.

A similar test was performed to quantify the shear detachment force as the module diameter increases. This experiment was performed with the same setup as described previously, properly modified in order to apply a tangential load on the mucoadhesive module, as represented in Figure 4.

A continuous translational motion was imposed to the slider tangentially to the tissue with a speed in a range of $0,7 \mathrm{~mm} / \mathrm{s}$ and the shear detachment force $\left(\mathrm{F}_{\mathrm{S}}\right)$ was determined. Also in this case, the best performing polymeric formulation was attached to cylindrical modules having different diameters (i.e. $12,15,20$ and $30 \mathrm{~mm}$ ). The preload force and time adopted in this experiment are the best ones assessed during the previous test sessions.

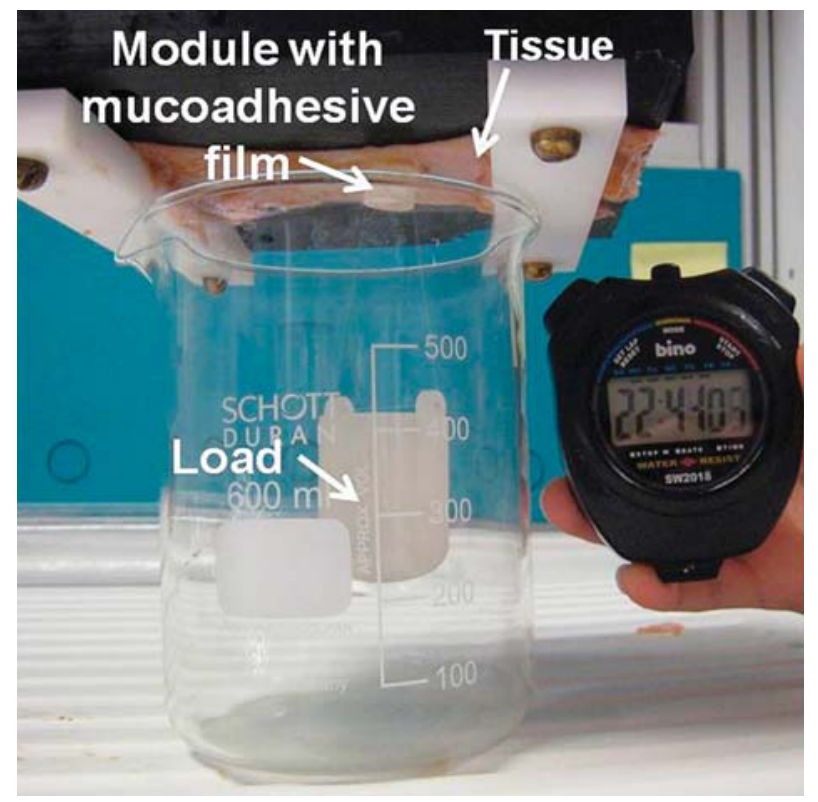

Figure 2. Experimental setup for debonding time measurement. 


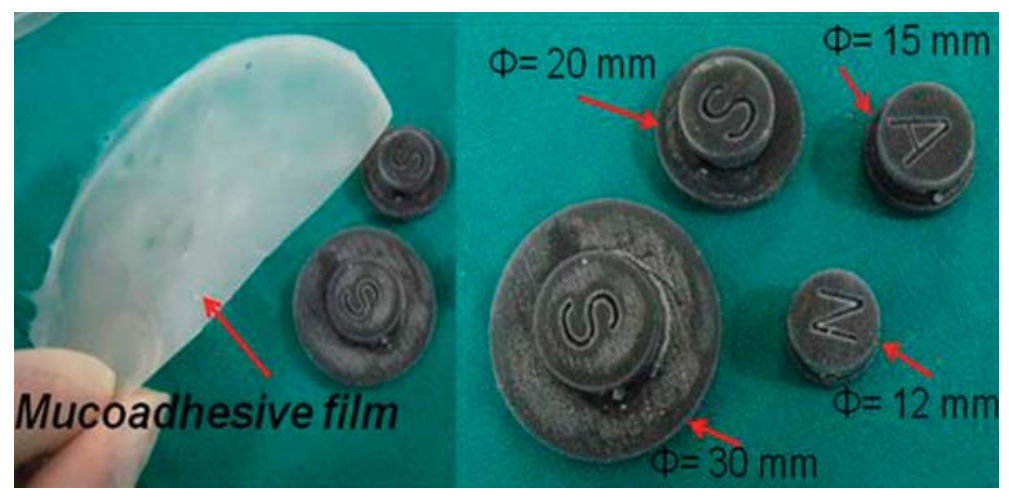

Figure 3. Cylindrical prototyping modules with 12, 15, 20 and $30 \mathrm{~mm}$ diameter.

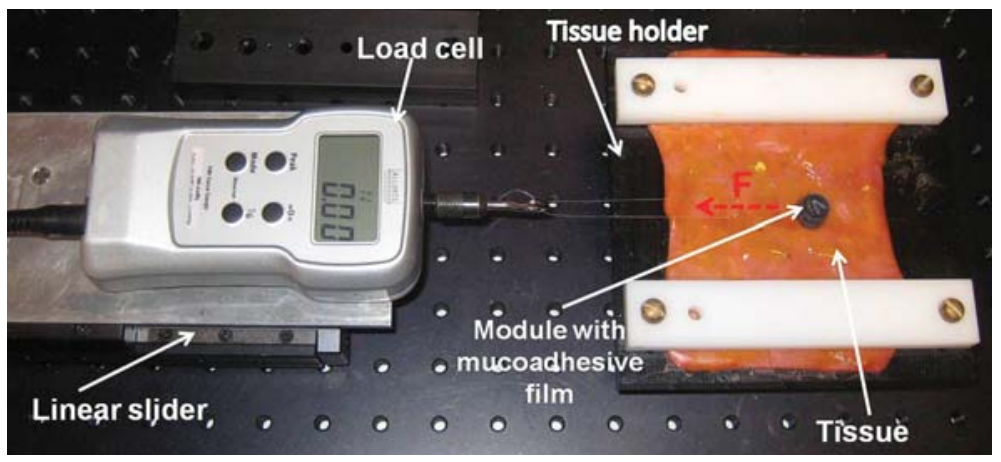

Figure 4. Experimental setup for shear test.

A final test was performed with a $30 \mathrm{~mm}$ diameter module, featuring the S3 formulation, the optimal preload force and time and a weight of $45 \mathrm{~g}$. The goal of this experiment was to assess the performance of the largest surface still compatible with an SPL procedure while supporting the same weight of the AISI reported by Cadeddu and coworkers (2).

\section{Results and discussion}

In vitro swelling studies

The hydration process requires a precise analysis in terms of time, because the hydration of a bioadhesive polymer is essential to initiate the mucoadhesive bonding process. For this objective, water uptake study and swelling index relate the water content to the mechanical properties of the different mucoadhesive formulations.

Observing the initial period (e.g. up to two minutes), Figure 5 shows that S2, S3 and S4 exceed a swelling index value of 2 in two minutes; this almost instantaneous uptake of water favors the hydrogen bonding between mucous and polymer and is thus crucial for initiating the adhesion. This behavior is consistent with the differences in the mucoadhesive chemical formulations, because higher values of swelling index were found in formulations with increased concentration of Carbopol (the best value were obtained firstly by $\mathrm{S} 4$, and then by $\mathrm{S} 2$ and $\mathrm{S} 3$ ).

The dynamic uptake of water is also an important parameter of the polymeric system. Considering the characteristic curves of the four samples, and in particular the values reached after 15 minutes, we can observe that swelling indexes of S3 and S4 grow significantly up to 4 , while S2 and S1 presented a lower slope, which can be ascribed to their lower content of Carbopol (0,3\%). In this case, formulations with a limited water absorption at long term - at 15 minutes- have to be preferred because this suggests a higher stability.

From a comparative study of the swelling index values and profiles of the four formulations, S2 and S3 formulations represent the most suitable samples for our application as best tradeoff between the short and long term behaviors. 


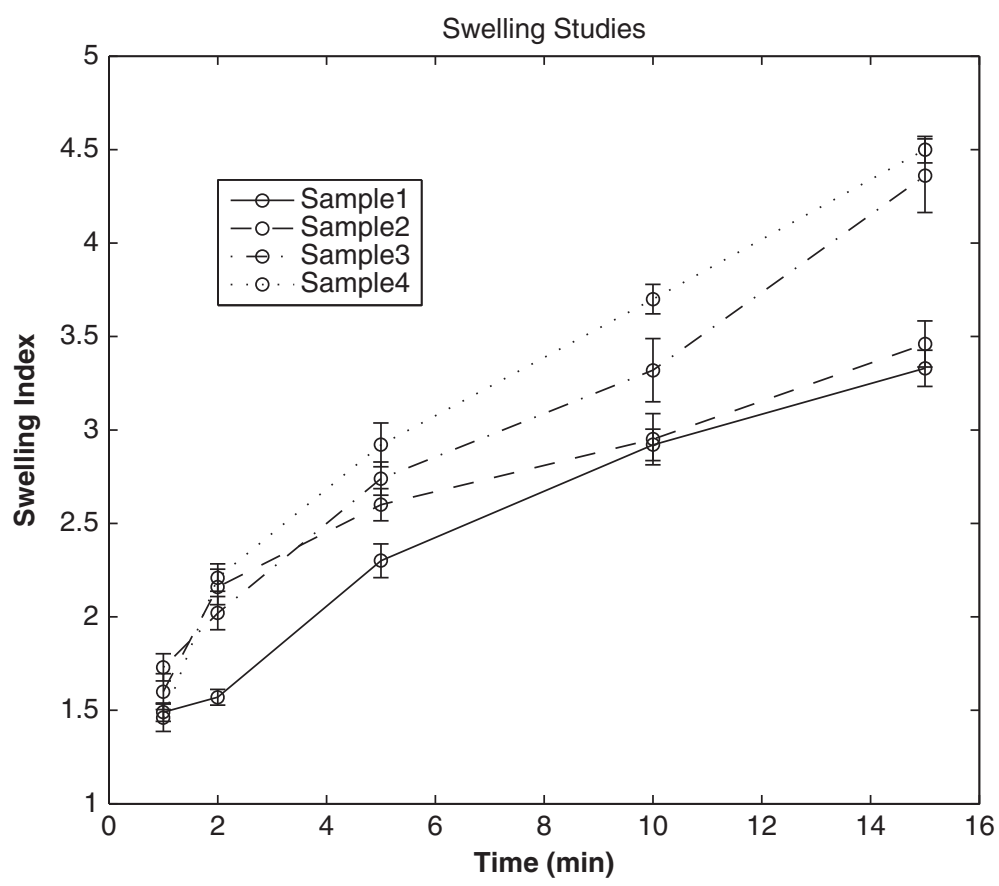

Figure 5. Comparative study of the swelling index profiles of S1, S2, S3 and S4 mucoadhesive formulations.

Ex vivo mucoadhesion testing

The $\mathrm{pH}$ value measured on the freshly excised porcine tissues was about 3 in low-mucous zone, reaching a maximum of 6 , depending on the presence of mucous.

\section{Adhesion performance and preload requirements}

The $F_{D}$ values required to remove the four mucoadhesive samples (S1, S2, S3, S4) from the porcine gastric tissue are collected in Table II.

The adhesion between mucoadhesive film and ex vivo gastric tissue was observed with the four chemical formulations reported in Table I. All the formulations showed mucoadhesive forces in the range of $1,32-2,63 \mathrm{~N}$ applying a preload force of $5 \mathrm{~N}$, and these values increased up to $1,51-2,81 \mathrm{~N}$ when a preload force of $10 \mathrm{~N}$ was applied. It is worth mentioning that an increase of about $0,1-0,2 \mathrm{~N}$ (i.e. the $6 \%$ of $F_{D}$ with $5 \mathrm{~N}$ preload) in the detachment force was observed by raising the preload force from $5 \mathrm{~N}$ to $10 \mathrm{~N}$.

In order to investigate the effect of speed on the detachment forces, the same test was repeated (data not shown) adopting a faster $(0,9 \mathrm{~mm} / \mathrm{s})$ and a slower $(0,5 \mathrm{~mm} / \mathrm{s})$ motion. The detachment force presented a variation of $1 \%$ over the full speed range, thus suggesting that the effect of speed on the ex vivo results was negligible.

Moreover, it can be observed from Table II that, given a value of preload force, $F_{D}$ for S3 and S4 films $(1 \% \mathrm{w} / \mathrm{w}$ Carbopol) were significantly greater than the forces required to detach S1 and S2 (0,3\% $\mathrm{w} / \mathrm{w}$ Carbopol), respectively. Finally, an increase in Carbopol concentration results in a significant raise in mucoadhesive strength $\left(F_{D}\right.$ for $S 3>F_{D}$ for $\left.S 1\right)$ due to the availability of several carboxylic groups that

Table II. Detachment force $\left(F_{D}\right)$ of the 4 different mucoadhesive formulations. Values are expressed as mean \pm S.D.

\begin{tabular}{|c|c|c|c|c|}
\hline \multirow{2}{*}{ Formulation Preload conditions } & \multicolumn{2}{|c|}{ Preload Force: $5 \mathrm{~N}$} & \multicolumn{2}{|c|}{ Preload Force: $10 \mathrm{~N}$} \\
\hline & 3 minutes & 5 minutes & 3 minutes & 5 minutes \\
\hline$S 1$ & $\mathrm{~F}_{\mathrm{D}}=1,43 \mathrm{~N} \pm 0,17$ & $\mathrm{~F}_{\mathrm{D}}=1,59 \mathrm{~N} \pm 0,31$ & $\mathrm{~F}_{\mathrm{D}}=1,70 \mathrm{~N} \pm 0,16$ & $\mathrm{~F}_{\mathrm{D}}=1,91 \mathrm{~N} \pm 0,21$ \\
\hline$S 2$ & $\mathrm{~F}_{\mathrm{D}}=1,32 \mathrm{~N} \pm 0,11$ & $\mathrm{~F}_{\mathrm{D}}=1,55 \mathrm{~N} \pm 0,2$ & $\mathrm{~F}_{\mathrm{D}}=1,51 \mathrm{~N} \pm 0,39$ & $\mathrm{~F}_{\mathrm{D}}=1,74 \mathrm{~N} \pm 0,11$ \\
\hline$S 3$ & $\mathrm{~F}_{\mathrm{D}}=2,62 \mathrm{~N} \pm 0,12$ & $\mathrm{~F}_{\mathrm{D}}=2,63 \mathrm{~N} \pm 0,14$ & $\mathrm{~F}_{\mathrm{D}}=2,64 \mathrm{~N} \pm 0,13$ & $\mathrm{~F}_{\mathrm{D}}=2,81 \mathrm{~N} \pm 0,11$ \\
\hline$S 4$ & $\mathrm{~F}_{\mathrm{D}}=1,66 \mathrm{~N} \pm 0,14$ & $\mathrm{~F}_{\mathrm{D}}=1,68 \mathrm{~N} \pm 0,12$ & $\mathrm{~F}_{\mathrm{D}}=1,60 \mathrm{~N} \pm 0,13$ & $\mathrm{~F}_{\mathrm{D}}=1,78 \mathrm{~N} \pm 0,15$ \\
\hline
\end{tabular}


determines bioadhesion between Carbopol and the mucous layer.

As reported by Lehr et al. (27), in the first phase the adhesion is favored by the swelling of the polymer, which comes into intimate contact with the mucous covered surface. In this contact, thanks to the exerted preload force, physical connections are established, i.e. there is the interpenetration of the polymeric chains of the film within the mucous's glycoproteic and olygosaccaridic chains. In this same step hydrogen bonds with sugar residues. This strengthens the mucous gel network and assures formulation adhesiveness for an extended period of time. Comparing the behavior of the different formulations, $\mathrm{S} 1$ and $\mathrm{S} 3$ showed greater mucoadhesive strength on gastric mucosa rather than S2 and S4, respectively. The addition of PPG (in $\mathrm{S} 1$ and S3) in the polymer preparation has thus to be preferred to PEG (S2 and S4). While PEG acts as plasticizer and varies the wettability of the film, the insertion of PPG can improve the film performance, not only strengthening the film structure and preventing any damage during demoulding and storage, but also adding $-\mathrm{OH}$ groups.

Based on experimental results, the best performance $\left(\mathrm{F}_{\mathrm{D}} \approx 3 \mathrm{~N}\right)$ was obtained with $\mathrm{S} 3$ formulation (1\% Carbopol, 3\% PPG) using a preload force of $10 \mathrm{~N}$ for five minutes. Considering that by increasing the preload force from $5 \mathrm{~N}$ up to $10 \mathrm{~N}$, a negligible increment in $\mathrm{F}_{\mathrm{D}}$ was obtained (around 6\%), $5 \mathrm{~N}$ of preload force was selected for the following experiments.

As regards the preload time, with $5 \mathrm{~N}$ of preload force, $F_{D}$ does not increase significantly between three and five minutes, as shown in Table II. A single minute of preload was also tested (data not shown), but it was not sufficient for establishing a stable bond between the polymeric film and the tissue. Considering that time is always a critical issue in surgical procedures, a preload of three minutes seems to be the best tradeoff to promote a stable adhesion in a reasonable timeframe.

Based on these considerations, a preload force of $5 \mathrm{~N}$ applied to the S3 mucoadhesive film for three minutes turns out to be the optimal configuration among the investigated ones.

\section{Measurements of sustainable weight and size}

The first trial of this session was aimed to measure the anchoring time guaranteed by the four different formulations as the load increases. The results are reported in Table III.

The tested formulations showed debonding time ranging from seven minutes up to one hour and
Table III. Debonding time versus total weight for the four formulations.

\begin{tabular}{llll}
\hline Load $(g)$ & \multicolumn{1}{c}{$15 \mathrm{~g}$} & \multicolumn{1}{c}{$20 \mathrm{~g}$} & \multicolumn{1}{c}{$25 \mathrm{~g}$} \\
\hline$S 1$ & $25 \mathrm{~min} 30 \mathrm{sec}$ & $15 \mathrm{~min} 43 \mathrm{sec}$ & $11 \mathrm{~min} 00 \mathrm{sec}$ \\
$S 2$ & $13 \mathrm{~min} 45 \mathrm{sec}$ & $9 \mathrm{~min} 56 \mathrm{sec}$ & $7 \mathrm{~min} 03 \mathrm{sec}$ \\
$S 3$ & $1 \mathrm{~h} 14 \mathrm{~min}$ & $1 \mathrm{~h} 03 \mathrm{~min}$ & $47 \mathrm{~min} 29 \mathrm{sec}$ \\
$S 4$ & $46 \mathrm{~min} 30 \mathrm{sec}$ & $40 \mathrm{~min} 18 \mathrm{sec}$ & $30 \min 37 \mathrm{sec}$ \\
\hline
\end{tabular}

15 minutes when a preload force of $5 \mathrm{~N}$ was applied for three minutes before the test. Also in this case S3 showed the best performance. Whether this operative lifetime is acceptable or not depends on the specific application the AISI is designed to perform.

In order to propose the mucoadhesion as positioning and anchoring strategy for different AISI, a relationship between $\mathrm{F}_{\mathrm{D}}$ and mucoadhesive module diameter was experimentally evaluated.

As shown in Table IV, $\mathrm{F}_{\mathrm{D}}$ grows rapidly from 2,6 to $12,1 \mathrm{~N}$ as the contact area increases from $113 \mathrm{~mm}^{2}$ up to $706 \mathrm{~mm}^{2}$. Confirming our hypothesis, the following equation applies to the experimental results:

$$
F_{D}=C_{D} r_{m o d}^{2}
$$

where $F_{D}$ is the achievable detachment force, $r_{\text {mod }}$ is the radius of the AISI adhesive surface and $C_{D}$ $\left(0,056 \pm 0,003 \mathrm{~N} / \mathrm{mm}^{2}\right)$ is an experimental coefficient relating $r_{\text {mod }}$ to $F_{D}$.

Thanks to this result, a tailored mucoadhesive area dimension can be designed given the weight of the AISI.

As regards the measurement of the shear detachment force $F_{S}(N)$, the $S 3$ formulation was used and a $5 \mathrm{~N}$ preload force was applied for three minutes to each sample under test. The results are collected in Table V.

As reported in Table $\mathrm{V}, \mathrm{F}_{\mathrm{S}}$ is $0,9 \mathrm{~N}$ for a module having $12 \mathrm{~mm}$ in diameter and this value reaches $4,4 \mathrm{~N}$ when the diameter increases from $12 \mathrm{~mm}$ up to $30 \mathrm{~mm}$. Also in this case the shear detachment force

Table IV. $\mathrm{F}_{\mathrm{D}}$ and module diameter relationship using $\mathrm{S} 3$ formulation with a preload force of $5 \mathrm{~N}$ applied for 3 minutes. Values are expressed as mean \pm S.D.

\begin{tabular}{lr}
\hline Module diameter $(\mathrm{mm})$ & $\mathrm{F}_{\mathrm{D}}(\mathrm{N})$ \\
\hline 12 & $2,6 \pm 0,1$ \\
15 & $2,9 \pm 0,1$ \\
20 & $4,9 \pm 0,13$ \\
30 & $12,1 \pm 0,1$ \\
\hline
\end{tabular}


Table V. Shear force versus module diameter for S3 mucoadhesive formulation. Values are expressed as mean \pm S.D.

\begin{tabular}{lr}
\hline Module diameter $(\mathrm{mm})$ & \multicolumn{1}{c}{$\mathrm{F}_{\mathrm{S}}(\mathrm{N})$} \\
\hline 12 & $0,9 \pm 0,2$ \\
15 & $1,2 \pm 0,1$ \\
20 & $3 \pm 0,3$ \\
30 & $4,4 \pm 0,3$ \\
\hline
\end{tabular}

grows proportionally to the mucoadhesive surface; therefore the following relation applies:

$$
F_{S}=C_{S} r_{m o d}^{2}
$$

where the experimental coefficient relating the shear detachment force to the module radius is $C_{S}=0,022 \pm$ $0,001 \mathrm{~N} / \mathrm{mm}^{2}$.

It is worth mentioning that the values related to detachment collected in the Tables II, IV, and V correspond to the force value before the peeling phase starts. This was confirmed by observing the shear force during the test. In particular, while the mucoadhesive module was attached onto the tissue, the force grew until a peak value was reached. Then, the force decreased due to the peeling phenomenon that induced the progressive detachment of mucoadhesive film from the gastric tissue.

In the final test, a $30 \mathrm{~mm}$ diameter module (contact area of $706 \mathrm{~mm}^{2}$ ) was able to support a weight of $45 \mathrm{~g}$ for 1 hour and 36 minutes by using S3 formulation and $5 \mathrm{~N}$ of preload force for three minutes.

\section{Conclusion}

In this paper mucoadhesion is proposed as anchoring mechanism for assistive surgical instrumentation to be deployed into the gastric cavity by means of scarless surgical procedures. In order to prove this concept, a mucoadhesive formulation was investigated and validated in vitro and ex vivo on freshly excised swine stomach tissue for attaching modules with different weight and dimension.

As regards the choice of the materials for preparing the film, several criteria were considered. Mucoadhesive formulations are widely used in pharmaceutics for designing drug delivery systems. In this case, as summarized in (18), the main criteria to be taken into account are related to the rate and period of drug delivery, the structural characteristics of tissue and polymer, and the target area for treatment. On the other hand, for the presented strategy of AISI anchoring, the materials must be selected in order to achieve satisfactory performance in terms of duration and stability (swelling time), and of adhesion force (detachment and shear force and stress). Moreover, the designed polymer must be synthesized in film shape.

Four variations were derived from the formulation presented by Dodou et al. for the colon (19), by keeping the active component of the mucoadhesive film and varying the concentrations of binder and plasticizer only. Observing the swelling behaviour, we selected the formulations (S2: 0,3\% Carbopol and 2\% PEG; S3: $1 \%$ Carbopol) which showed the higher uptake of water after two minutes and the lower value of hydratation after 15 minutes. These characteristics of the polymer favor the initial contact between film and mucous and a higher stability in the subsequent adhesion.

With a dedicated test bench, the preload conditions for gaining a stable attachment on mucous covered tissues were investigated. A preload force of $5 \mathrm{~N}$ must be exerted for three minutes in order to stick a module on the inner side of the gastric wall. Given this preload condition, the best performing formulation in terms of detachment force was S3. For a contact area of $113 \mathrm{~mm}^{2}$ (12 $\mathrm{mm}$ in diameter), the maximum normal and shear detachment forces withstood by the adhesive film are 2,6 $\mathrm{N}$ and $0,9 \mathrm{~N}$ respectively. These values grow to $12,14 \mathrm{~N}$ and $4,4 \mathrm{~N}$ as the contact area increases to $706 \mathrm{~mm}^{2}$ (30 mm diameter). As regards lifetime of the bonding, a $25 \mathrm{~g}$ module featuring a $12 \mathrm{~mm}$ diameter (contact area of $113 \mathrm{~mm}^{2}$ ) was able to resist against its own weight for 48 minutes. By increasing the module diameter up to $30 \mathrm{~mm}$ (contact area of $706 \mathrm{~mm}^{2}$ ), anchoring of a $45 \mathrm{~g}$ module - as the AISI reported in (2) - was achieved for 1 hour and 36 minutes.

Given that the adhesion is essentially a surface phenomenon, a linear relationship between normal and tangential detachment forces and the mucoadhesive contact area was confirmed by the ex vivo results. This allows to predict the required module diameter, given the AISI weight.

In conclusion, the proposed anchoring method was demonstrated to be a viable solution to fix AISI inside the gastric cavity from a single endoluminal access, overcoming the main limitations of using magnets. Despite these encouraging results, there are some important limitations that must be commented. In particular, other parameters could influence the mucoadhesive performance, such as the $\mathrm{pH}$ of the environment and the thickness of the film. These aspects will be addressed in future works. The procedure for deployment of mucoadhesive AISI in the stomach will also be defined, considering the results obtained so far in terms of preload force and time. 
In vivo tests will be performed to prove this solution also in a living physiological environment.

Now that the feasibility of this approach has been demonstrated for the stomach and preliminary experience has been gained from the procedural standpoint, future efforts should be devoted to extend this solution to other cavities of the human body, such as the abdomen, thus widening the impact on laparoscopic procedures. Even though the mucoadhesive film presented in this study could work on different anatomical districts, thanks to wet conditions, this does not imply that the proposed formulation is the best choice. Alternative polymers, which can lead to even better bonding performances in terms of duration and strength, should be considered, also depending on the peculiar AISI function. Additional in vitro, ex vivo and in vivo tests must be performed to select the best option in terms of chemical formulation, film thickness and interaction with tissue.

It is worth mentioning that the mucoadhesive formulation can be modified in order to include drugs that can be released locally on the tissues during and after the operative procedure. Moreover, magnetic micro or nanoparticles can be embedded in the film matrix, adding magnetic controllability to the bioadhesive behavior. This would enable the retraction of small organs, such as gall bladder, during a cholecystectomy. Thanks to magnetic loading of the polymeric structure and the intimate contact between the tissue and the film, additional surgical tasks could be performed, such as magnetic mucosal surface retraction or local hyperthermic therapy (31).

\section{Acknowledgment}

This material is based in part upon work supported by the European Commission in the framework of ARAKNES FP7 European Project 224565. The authors wish to thank N. Funaro, A. Melani, G. Favati and C. Filippeschi for prototypes manufacturing as well as Dr. Di Sacco for providing freshly excised porcine tissues.

Declaration of interest: The authors report no conflicts of interest. The authors alone are responsible for the content and writing of the paper.

\section{References}

1. Romanelli JR, Earle DB. Single-port laparoscopic surgery: an overview. Surg Endosc. 2009;23:1419-27.

2. Cadeddu J, Fernandez R, Desai M, Bergs R, Tracy C, Tang SJ, et al. Novel magnetically guided intra-abdominal camera to facilitate laparoendoscopic single-site surgery: initial human experience. Surg Endosc. 2009;23:1894-9.

3. Park S, Bergs RA, Eberhart R, Baker L, Fernandez R, Cadeddu JA. Trocar-less Instrumentation for Laparoscopy Magnetic Positioning of Intra-abdominal Camera and Retractor. Annals of Surgery 2007;245:379-84.

4. Scott DJ, Tang SJ, Fernandez R, Bergs R, Goova MT, Zeltser I, et al. Completely transvaginal NOTES cholecystectomy using magnetically anchored instruments. Surg Endosc. 2007;21:2308-16.

5. Lehman AC, Berg KA, Dumpert J, Wood NA, Visty AQ, Rentschler ME, et al. Surgery with cooperative robots. Computer Aided Surgery 2008;13:95-105.

6. Berg KA. Robot design and abdominal wall modeling for in vivo surgery. Thesis presented to the faculty of "the graduate college at the University of Nebraska", Dec. 2007.

7. Canes D, Lehman AC, Farritor SM, Oleynikov D, Desai MM. The future of NOTES instrumentation: flexible robotics and In vivo minirobots. Journal of endourology 2009; 23:787-92.

8. Ryou M, Thompson CC. Magnetic retraction in natural-orifice transluminal endoscopic surgery (NOTES): addressing the problem of traction and countertraction. Endoscopy 2009; 41:143-8.

9. Kaehler G, Grobholz R, Langner C, Suchan K, Post S. A new technique of endoscopic full-thickness resection using flexible stapler. Endoscopy 2006;38:86-9.

10. Pleskow D, Rothstein R, Haber G, Gostout C, Lo S, Hawes R, et al. Endoscopic full-thickness placation for the treatment of GERD: five-year long-term multicenter results. Surg Endosc. 2008;22:326-32.

11. Blackburn GL, Mun EC. Therapy insight: weight-loss surgery and major cardiovascular risk factor, Nat Clin Pract Cardiovasc Med. 2005;2:585-91.

12. Kobiela J, Stefaniak T, Mackowiak M, Lachinski AJ, Sledzinski Z. NOTES-third generation surgery, Vain hopes or the reality of tomorrow? Langenbecks Arch Surg. 2008;393: 405-11.

13. Harada K, Oetomo D, Susilo E, Menciassi A, Daney D, Merlet JP, et al. A reconfigurable modular robotic endoluminal surgical system: vision and preliminary results. Robotica [in press, available online].

14. Kwiatek MA, Pandolfino JE. The Bravo(TM) pH capsule system. Digestive and liver disease 2008;40:156-60.

15. Schostek S, Müller V, Rieber F, Ho CN, Rakoschi C, Anhoeck G, et al. Implantable optical sensor concept for the detection of gastrointestinal bleeding. Conference of the society for medical innovation and technology, SMIT 2008.

16. Errachid A, Ivorra A, Aguilo J, Villa R, Zine N, Bausells J. New technology for multi-sensor silicon needles for biomedical applications. Sens. Actuators B 2001;78:279-84.

17. Majithiya RJ, Raval AJ, Umrethia ML, Ghosh PK, Murthy RSR, Zeltser I, et al. Mucoadhesion enhancement: enhancement of mucoadhesion by blending anionic, cationic \& nonionic polymers. Drug Delivery Technology 2008;8: $40-5$.

18. Kharenko EA, Larionova NI, Demina NB. Mucoadhesive drug delivery system (Review). Pharmaceutical Chemistry Journal 2009;43:21-9.

19. Dodou D, Breedveld P, Wieringa PA. Stick, unstick, restick sticky films in the colon. Minim Invasiv Ther \& Allied Technol. 2006;15:286-95.

20. Dodou D, Breedveld P, Wieringa PA. Friction manipulation for intestinal locomotion. Minim Invasiv Ther \& Allied Technol. 2005;14:188-97. 
21. https://www.usendoscopy.com/Advance.php.

22. https://www.karlstorz.com/cps/rde/xchg/SID-ED98E43ED24FFE62/karlstorz-en/hs.xsl/8870.htm.

23. Dodou D, Aranzazu, del Campo, Arzt E. Mucoadhesive micropatterns for enhanced grip, IEEE EMBS Lyon, 2007.

24. Peppas NA, Bures P, Leobandung W, Ichikawa H. Hydrogels in pharmaceutical formulations. European Journal of Pharmaceutics and Biopharmaceutics 2000;50:27-46.

25. Peppas Na, Little MD, Huang Y. Handbook of Pharmaceutical Controlled Release Technology. 2001.

26. Robinson JR, Lee JW, Park JH. Bioadhesive-based dosage forms: the next generation. European Journal of Pharmaceutics and Biopharmaceutics 2000,89:850-66.

27. Lehr CM, Bouwstra JA, Spies F, Onderwater J, Noordeinde J, Vermeij-Keers C, et al. Visualization of the mucoadhesive interface. Journal of controlled release 1992; 18:249-60.

28. Harding SE, Davis SS, Deacon MP, Fiebrig I. Biopolymer mucoadhesives. Biotechnology and genetic engineering reviews 1999;16:41-86.

29. Efentakis M, Hatzi EC, Andreopoulos AG. Development, evaluation and release characteristics in vitro of oral mucosal bioadhesive matrices containing tetracycline for application to the oral cavity. STP pharma sciences 1998;8:227-32.

30. Dodou D, Colonic locomotion. PhD-thesis, Delft University of Technology, Netherlands, Sept 2006.

31. Wang $Z$, Wang $L$, Tang $B$, Frank $T$, Brown $S$, Cuschieri A. Retraction by surface ferromagnetisation of target tissues: Preliminary studies on feasibility of magnetic retraction for endoscopic surgery. Surg Endosc. 2008;22:1838-44. 